\title{
Cluster view of the plasma sheet boundary layer and bursty bulk flow connection
}

\author{
O. W. Lennartsson ${ }^{1}$, L. M. Kistler ${ }^{2}$, and H. Rème ${ }^{3}$ \\ ${ }^{1}$ Advanced Technology Center, Lockheed Martin Space Systems Co., Palo Alto, CA 94304, USA \\ ${ }^{2}$ Space Science Center, University of New Hampshire, Durham, NH 03824, USA \\ ${ }^{3}$ CESR/CNRS, BP 4346, 31028 Toulouse Cedex 4, France
}

Received: 20 January 2009 - Accepted: 27 March 2009 - Published: 8 April 2009

\begin{abstract}
The high-latitude boundaries of the plasma sheet (PSBL) are dynamic latitude zones of recurring and transient (minutes to tens of minutes) earthward and magnetic fieldaligned bursts of plasma, each being more or less confined in longitude as well, whose ionic component is dominated by protons with flux, energies and density that are consistent with a central plasma sheet (CPS) source at varying distance (varying rates of energy time dispersion), sometimes as close as the $\sim 19 R_{E}$ Cluster apogees, or closer still. The arguably most plausible source consists of so called "bursty bulk flows" (BBFs), i.e. proton bulk flow events with large, positive and bursty GSE $v_{x}$. Known mainly from CPS observations made at GSE $x>-30 R_{E}$, the BBF type events probably take place much further downtail as well. What makes the BBFs an especially plausible source are (1) their earthward bulk flow, which helps explain the lack of distinctive latitudinal PSBL energy dispersion, and (2) their association with a transient strong increase of the local tail $B_{z}$ component ("local dipolarization"). The enhanced $B_{z}$ provides intermittent access to higher latitudes for the CPS plasma, resulting in local density reductions in the tail midplane, as illustrated here by proton data from the Cluster CIS CODIF instruments. Another sign of kinship between the PSBL bursts and the BBFs is their similar spatial fine structure. The PSBL bursts have prominent filaments aligned along the magnetic field with transverse flux gradients that are often characterized by local $\sim 10 \mathrm{keV}$ proton gyroradii scale size (or even smaller), as evidenced by Cluster measurements. The same kind of fine structure is also found during Cluster near-apogee traversals of the tail midplane, as illustrated here and implied by recently published statistics on BBFs obtained with Cluster multipoint observations at varying satellite separations. Altogether, the Cluster observations
\end{abstract}

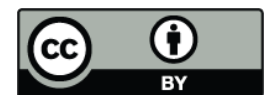

Correspondence to: $\mathrm{O} . \mathrm{W}$. Lennartsson (lenn@mailhost.spasci.com) described here mesh rather well with theories about so called plasma sheet "bubbles," i.e. earthward drifting closed magnetic flux tubes with reduced particle pressure and enhanced magnetic field strength at their apex. It is argued that such bubbles may be initiated by localized diamagnetic instabilities.

Keywords. Magnetospheric physics (Magnetospheric configuration and dynamics; Magnetotail; Plasma sheet)

\section{Introduction}

The principal defining feature of the so called "plasma sheet boundary layer," or PSBL for short, has been its location, being observed immediately adjacent to a tail lobe, usually as a rather brief $(<20 \mathrm{~min})$ enhancement of the energetic $(\mathrm{keV})$ particle flux. In addition, some proton velocity or energy dispersion is often noted, along with initially earthward bulk motion (e.g. Parks et al., 1979, 1984; Forbes et al., 1981; Spjeldvik and Fritz, 1981; Williams, 1981; Eastman et al., 1985; Bosqued et al., 1993). From the first observations, this phenomenon has been commonly viewed as a manifestation of a "neutral line" (or "X-line") being located tailward of the observing spacecraft.

For example, Forbes et al. (1981), using particle data from the near-equatorial ISEE 1 and ISEE 2 satellites, reported observing several events of protons streaming along the magnetic field near the northern surface of the plasma sheet, each event having protons streaming only earthward at first, and then both earthward and tailward, the latter identified as mirrored protons. In the one case shown, from about 15 Earth radii $\left(R_{E}\right)$ downtail, the mirrored protons appear about $2 \mathrm{~min}$ after the arrival of earthward-moving protons. The speeds of both populations show a systematic decrease with time, which is interpreted as due to the plasma source moving tailward onto field lines mapping to progressively higher polar

Published by Copernicus Publications on behalf of the European Geosciences Union. 
latitudes (above the satellites). The authors suggest that the plasma source is spatially associated with a tailward retreating neutral line.

An essential consequence of this interpretation is that the PSBL proton energies must have a continuous latitudinal dispersion, with the average energy increasing with increasing latitude. This is true of any "customary" neutral-line source model, whether that source moves tailward or not, because a tail neutral line, by definition, must be immersed in a duskdirected large-scale electric field, where the associated earthward and equator-directed $\boldsymbol{E} \times \boldsymbol{B}$ drift will disperse ions according to their magnetic field-aligned velocity as they all drift earthward, away from the source. Its reverse implication, which is decreasing proton energy with decreasing latitude, is often invoked in the literature, when proton energies are observed to decrease with time, to argue that the observing spacecraft is becoming engulfed by the central plasma sheet (CPS).

In an attempt to separate latitudinal and temporal dispersion effects, Lennartsson et al. (2001) applied ion spectral data from the polar orbiting Polar satellite to compare a large number of northward (autumn) and southward (spring) crossings of the night-side boundary between the plasma sheet and the northern lobe at geocentric distances $R \sim 4$ to $7 R_{E}$. Their ion data, in the form of differential flux versus energy and time, were obtained with the TIMAS instrument (toroidal imaging mass-angle spectrograph) at energy per charge between $15 \mathrm{eV} / \mathrm{e}$ and $33 \mathrm{keV} / \mathrm{e}$ at a time resolution of $12 \mathrm{~s}$ (two $\mathrm{s} / \mathrm{c}$ spin cycles). This comparison revealed that individual proton energy dispersion traces, when clearly defined, which occurred on about $60 \%$ of the crossings, slope downward as function of time, whether the ion energy analyzer is moving poleward or equatorward.

Of a total of 106 poleward crossings, none displayed a distinct continuous increase, even briefly, of proton energy with time (Table 1 in Lennartsson et al.). The only upward trend found, during five of those crossings, consisted of multiple brief ( $\sim$ few minutes) flux enhancements at successively higher energy. Even during these "stepping up" events, the individual flux patches showed a downward energy trend (e.g. Plate 2 in the Lennartsson et al. paper).

These Polar results were later confirmed with ion statistical data from the polar orbiting Interball-Auroral satellite by Sauvaud and Kovrazhkin (2004), data taken at altitudes of 2 to $3 R_{E}$, and with some event data (at $R>4 R_{E}$ ) from the Cluster satellites as well (Sauvaud and Kovrazhkin, 2004; Keiling et al., 2004). The Sauvaud and Kovrazhkin study treats the stepping up kind of events as a separate type (VDIS), based in part on their occurring during reduced magnetic activity and in association with less energetic electrons. However, the downward energy trend within each proton substructure was confirmed.

As far as their energy dispersion is concerned, PSBL proton flows are thus consistent with transient bursts, probably from a transient but recurring kind of source. Given that a dawn-to-dusk electric field is still present, then the absence of latitudinal dispersion near Earth implies that the tail plasma source is moving earthward itself. That is, as the burst plasma expands earthward along magnetic field lines, all plasma in a given magnetic flux tube, including the source itself, is convecting earthward (Lennartsson et al., 2001).

According to the Polar TIMAS survey, these PSBL proton bursts, within the few hours of midnight magnetic local time (MLT) that were examined, have differential number flux, energies, and number density that are consistent with a central plasma sheet source. The flux typically peaks at $10^{5}-$ $10^{6}\left(\mathrm{~cm}^{2} \mathrm{sr} \mathrm{s} \mathrm{keV}\right)^{-1}$ at an energy of $4-12 \mathrm{keV}$, like in the plasma sheet, and its pitch-angle distribution, being somewhat "field aligned" at first, is consistent with time-of-flight separation of an isotropic source population. The flux energy dispersion is generally more pronounced than its pitch-angle dispersion at the Polar altitudes, being a combination of fading flux at higher energy, as higher-energy protons mirror and return tailward, and increasing flux at lower energy, as successively lower-energy protons arrive $\left(R\right.$ being $\left.\leq 7 R_{E}\right)$. Peak number densities are usually in the same $0.1-1.0 \mathrm{~cm}^{-3}$ range as the central plasma sheet density.

The downward slope of the energy dispersion varies a great deal, and it is often steep enough (few minutes) for the source to be well earthward of $30 R_{E}$. It does not show a systematic ordering by latitude along the Polar orbit, however, and the most poleward flux structure sometimes has the steepest slope, consistent with its arriving along a "dipolarized" magnetic flux tube of limited extent in both latitude and longitude (e.g. Plate 3 in the Lennartsson et al. paper). The bursts tend to be more numerous, intense and energetic and to occur over a wider latitude range (up to five or more degrees invariant latitude along the Polar orbit) during high global geomagnetic activity, but they do occur at all levels of $K_{p}$, for instance, possibly being strongly localized at times. Some form of local proton flux enhancement, with or without clear dispersion, is seen on most of the Polar crossings of the (northern) plasma-sheet tail-lobe boundary.

It is not a long stretch of the imagination to associate such a convecting central plasma sheet source with the so called "bursty bulk flow events," or BBFs, i.e. proton bulk flow events with large, positive and bursty GSE $v_{x}$ (e.g. Baumjohann et al., 1990; Angelopoulos et al., 1992, 1994, 1997; Petrukovich et al., 2001; Raj et al., 2002; Ohtani et al., 2004; Cao et al., 2006). These near-midplane bursty flows, inferred from velocity moments, are mainly perpendicular to the local magnetic field, but they are accompanied by locally enhanced northward $B_{z}$, or local dipolarization, which allows latitudinal (and earthward) expansion of the plasma by fieldaligned flows, both northward and southward, with little or no net $z$ component of flow velocity. Such poleward expansion is probably what causes the transient reduction in density that is statistically associated with the transient dipolarization (Ohtani et al., 2004). Again, while this poleward plasma expansion is taking place, it does not necessarily 
contribute to bulk flow in the midplane, because of northsouth symmetry.

A physical connection between the equatorial BBFs and high-latitude field-aligned particle flows has already been alluded to in the literature (e.g. Sergeev et al., 2000a, b; Petrukovich et al., 2001), but arguments have been made against it as well (Raj et al., 2002). Arguments in favor of a connection must rely on the assumption that BBFs observed at GSE $x>-30 R_{E}$ are representative of such events much further downtail, in order to explain the common observation of PSBL structures with enhanced proton flux, with or without distinct dispersion, by near-Earth polar orbiting satellites ( $>50 \%$ ). According to Ohtani et al. (2004), all major BBF characteristics can be found irrespective of the $x$ distance between $x=-5$ and $-31 R_{E}$ and are found with increasing frequency in the tailward direction (their Fig. 1).

A large fraction of the plasma sheet-lobe boundary crossings in both the Polar (Lennartsson et al., 2001) and the Interball-Auroral (Sauvaud and Kovrazhkin, 2004) surveys, about $40 \%$ and $50 \%$, respectively, did not reveal distinct energy dispersion in the proton spectra. One important reason, as deduced from the Polar TIMAS spectra, is that the proton flux and number density often have very steep gradients transverse to the magnetic field lines, with scale sizes comparable to local $\mathrm{keV}$ proton gyroradii. As a consequence, only the most energetic protons, the ones with the largest gyroradii, may at times intersect the instrument, while at other times an entire proton filament may drift across the instrument too rapidly to reveal the dispersion of fieldaligned speed. These steep gradients have been confirmed with multipoint measurements by the Cluster CODIF instruments (composition and distribution function). Specifically, Lennartsson et al. (2007) show that the proton density with $40 \mathrm{eV} \leq E \leq 40 \mathrm{keV}$ can vary by $\Delta n=0.4 \mathrm{~cm}^{-3}$ across less than five average $90^{\circ}$ pitch angle proton gyroradii at $R \approx 5 R_{E}$ (average $E \approx 7.5 \mathrm{keV}$ at the time). See also the Cluster CODIF measurements of "beamlet" sizes at greater distance by Grigorenko et al. (2007), as well as the early ISEE-1 observations by Huang et al. (1987), which compared fluxes of $24-65 \mathrm{keV}$ protons with gyro centers on opposite sides of the satellite.

Similar fine structure is indeed also found with the BBF events, when these are observed by the Cluster satellites near their equatorial apogees at about $19 R_{E}$. A first Cluster statistical investigation of the cross-section widths of high-speed transverse flows was undertaken by Nakamura et al. (2004), using three-point CODIF velocity moments from the July to October 2001 period. Although the nominal maximum satellite separation was only about $2000 \mathrm{~km}$ at the time, they estimate the full widths of the flow channel to be $2-3 R_{E}$ in the GSM $x-y$ plane and $1.5-2 R_{E}$ along the GSM z-axis by assuming a triangular profile for $|\boldsymbol{v}(\boldsymbol{r})|$ in both directions, with the peak of each triangle defined by the satellite having the largest value of $\left(v_{\perp x}^{2}+v_{\perp y}^{2}\right)^{1 / 2}$. The authors do caution, however, that their linear extrapolations may not be realistic, and that the flow velocity profile could, for instance, have a small gradient at the center of the flow and fall off sharply at the edges.

This caveat is judicious, given the subsequent statistical results of Cao et al. (2006), who employ the same threepoint CODIF velocity moments but include data from 2002, as well. Their intent was to compare the probability of observing BBFs with three sampling points, given some criteria on the moments, to that probability with only a single sampling point (only SC1). The inclusion of year $2002 \mathrm{ob}-$ servations makes a remarkable difference. Specifically, the results from 2001, with separations of about $2000 \mathrm{~km}$, show only $2 \%$ more BBFs with all three satellites (137 events) than with one (134 events), while in 2002, with separations of about $4000 \mathrm{~km}$, twice as large, this ratio is almost threefold, or $257 \%$ (72 versus 28 events). In other words, with $0.6 R_{E}$ separation, the three satellites observe almost entirely different events. These numbers were obtained with the Angelopoulos et al. (1994) BBF criteria, but the other criteria are said to give similar results.

The Cao et al. results are strong evidence that the BBFs, like the high-latitude proton bursts, have spatial scale sizes that compare with local proton gyroradii. The tail magnetic field strength being typically $\leq 20 \mathrm{nT}$ near the Cluster apogees, $2000 \mathrm{~km}$ is at most three times larger than the gyroradius of, say, a $10 \mathrm{keV}$ proton at $90^{\circ}$ pitch angle. Actually, the satellite separations perpendicular to a given line are usually less than the nominal $2000 \mathrm{~km}$ envelope. Anyway, as the three observation points are separated beyond a couple of 10$\mathrm{keV}$ proton gyroradii (but not beyond six), in year 2002, the three concurrent CODIF proton flux measurements of Cao et al. clearly loose coherence, because of sharp spatial flux gradients. These sharp gradients would thus be expected in the associated poleward and field-aligned expanding plasma, as well.

Considering the 2002 Cao et al. statistics, it is very difficult, if not impossible, to obtain a meaningful conjugate comparison between a specific equatorial BBF flow burst and a concurrent high-latitude PSBL proton burst, especially since that comparison would require a s/c separation of several $R_{E}$ in GSE (and GSM) $x$ and $z$ (not available with Cluster alone). A more practical approach toward establishing a probable physical connection between the two kinds of flow is to identify recurrent similarities. Some such similarities have been pointed out above with regard to their respective (1) differential flux, (2) dynamics, and (3) spatial fine structure. The purpose of this report is to make these similarities more tangible by showing some representative proton and magnetic field data from the Cluster satellites.

\section{Cluster CODIF instruments}

The Cluster Ion Spectrometry (CIS) experiment consists of three identical sets of two instruments each, the CODIF, 


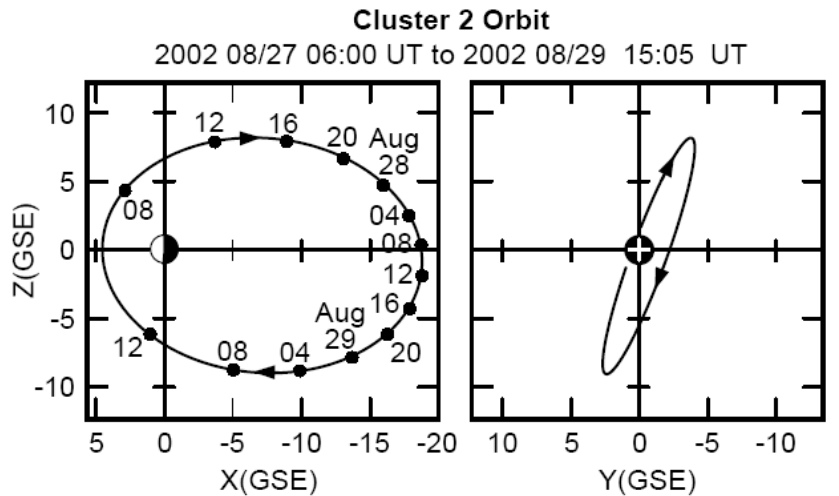

Fig. 1. Cluster orbit in geocentric solar ecliptic (GSE) coordinates (units are Earth radii, $R_{E}$ ): (left) $\mathrm{x}-\mathrm{z}$ projection with time labels at four-hour intervals. (right) y-z projection, sunward view (adapted from http://pwg.gsfc.nasa.gov/cgi-bin/gif_walk).

or Composition and Distribution Function analyzer, and the HIA, or Hot Ion Analyzer. There were originally four sets of instruments, one on each of the four Cluster satellites, but only three were successfully activated on orbit. These are on $\mathrm{SC} 1,3$ and 4. A comprehensive description of the instruments and their first measurements is given by Rème et al. (2001).

Very briefly, CODIF and HIA both have "top hat" kind of electrostatic energy analyzers, allowing for an $8^{\circ}$ by (almost) $360^{\circ}$ instantaneous field of view and $4 \pi$ angular coverage during each four second s/c spin cycle. HIA has the finer angular resolution but lacks the mass resolving power of CODIF, the latter using time-of-flight analysis. The present study is based entirely on CODIF measurements, supported by magnetic field data from the Cluster FGM instruments (Balogh et al., 2001).

In the case of CODIF, the $360^{\circ}$ range is divided into two sets of $22.5^{\circ}$ sectors, one set covering $180^{\circ}$ with higher sensitivity (GF. $\Delta E / E=1.9 \times 10^{-2} \mathrm{~cm}^{2} \mathrm{sr} \mathrm{keV} / \mathrm{keV}$ ) and the other $135^{\circ}$ with lower sensitivity $\left(2.1 \times 10^{-4} \mathrm{~cm}^{2} \mathrm{sr} \mathrm{keV} / \mathrm{keV}\right)$, in order to obtain the required dynamic flux range. The 32 energy sweeps each spin cycle yield an effective angular resolution of about $\left(8^{\circ}+11.2^{\circ}\right) \times 22.5^{\circ}$. The normal energy range (without RPA sweeps) is $0.02-38 \mathrm{keV} / \mathrm{e}$, usually covered in 30 steps. Data shown here have been averaged over either view angle (energy spectra) or energy (pitch-angle spectra) or integrated over both (velocity moments). The data have an intrinsic time resolution of a single 4-s spin cycle, but are typically displayed as two-spin $(8 \mathrm{~s})$ averages, and occasionally as four-spin averages.

\section{Cluster orbits}

Figure 1 depicts two geocentric solar ecliptic (GSE) projections of a Cluster orbit with apogee in the central geomagnetic tail. The four satellites have slightly different orbits,
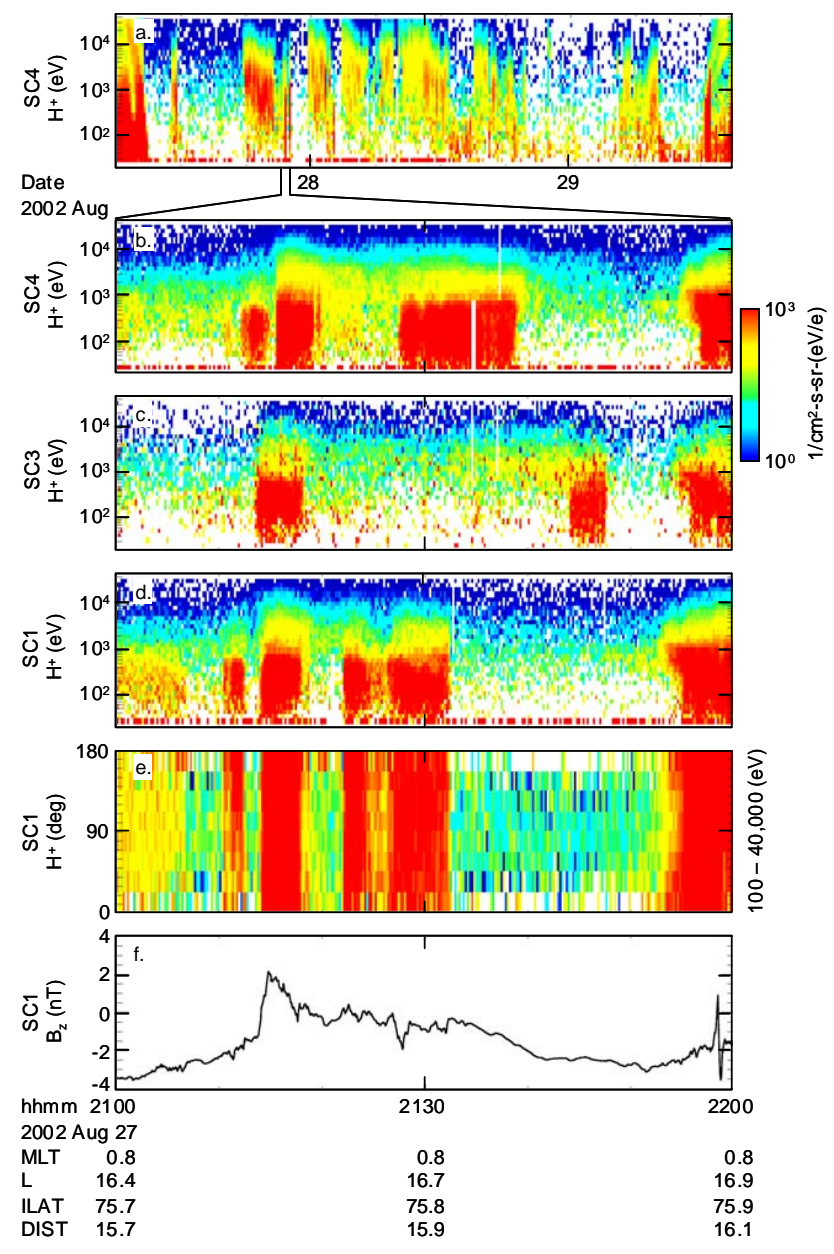

Fig. 2. (a) Spacecraft (SC) No. 4 differential proton flux, averaged over view angles $(4 \pi)$ and color coded (color bar on the right), versus time (x-axis) and energy (y-axis). (b-d) One-hour expanded views of flux from SC4, 3, and 1. (e) SC1 differential flux, averaged over energy $(0.1-40 \mathrm{keV})$ and sorted by pitch angle. (f) GSE $z$ component of magnetic field measured at SC1. Ephemeris at bottom is that of SC1 (cf. Table 1).

depending on the varying separation strategy. The orbit in Fig. 1 is that of SC2, crossing the nighttime x-y plane at GSE $x=-19.0 R_{E}$ and $y=-2.0 R_{E}$. The other satellites have their crossing points within $\Delta x= \pm 0.3 R_{E}$ and $\Delta y=0.5 R_{E}$ on this day, all crossing $z=0$ in the course of $55 \mathrm{~min}$. The orbital period of each satellite is about $57.1 \mathrm{~h}$.

\section{Near-midnight tail observations}

\subsection{7-29 August 2002}

Figure 2 displays data from within the time interval of Fig. 1. The top differential proton flux data, panel (a), averaged over view angles, represent an "ordinary" set from among the subset of orbits with very nearly uninterrupted data of equal 
two-spin resolution from all three CODIF instruments, although panel (a), spanning a complete orbit, only has data from SC4. The choice of SC4 is arbitrary here. These data are ordinary in appearance and also in the sense that current geomagnetic conditions are neither very active nor extremely quiet. The maximum three-hour $K_{p}$ during this interval is $3+$ between 21:00 and 24:00 UT on 27 August, and the 24-h $\Sigma K_{p}$ on 27, 28 and 29 August is $22,15-$ and 15, respectively.

\subsubsection{Plasma sheet filaments}

The occurrence in panel (a) of plasma sheet-like proton fluxes in multiple and seemingly isolated bundles, or "filaments" (Huang et al., 1987), is a feature common to all Cluster tail traversals, although the gaps between bundles are not always as wide as here. To reconcile this with the traditional image of the plasma sheet as a monolithic structure, one needs to keep in mind that it takes about $50 \mathrm{~h}$ for the satellites to travel through the tail from above the North Pole to below the South Pole (Fig. 1). That is ample time for the plasma distribution to undergo many changes. The multiple structures are in fact associated with both temporal and spatial variations. The temporal variations include the same kind of proton energy dispersion that is familiar from observations closer to Earth (e.g. Lennartsson et al., 2001; Sauvaud and Kovrazhkin, 2004) and will be described briefly below.

To highlight the often complex spatial aspects here, a onehour interval from near the end of 27 August is expanded in panel (b), and simultaneous data from SC3 and SC1 are shown in panels (c) and (d), respectively (bottom energy channel of SC1 and SC4 have spurious counts here). With the possible exception of the second and last structures in panel (b), the concurrent structures in panels (c) and (d) are different, both from panel (b) and from each other. The satellite separations at this time are listed in Table 1 (at 21:30 UT). These are examples of the commonly occurring local highlatitude proton flux enhancements without distinct energy dispersion, save for a hint of dispersion in the last structure, but they have lower than typical energies.

Panel (e) shows the flux pitch-angle distribution in the panel (d) structures, averaged over most of the energy range ( $40000 \mathrm{eV}$ is a rounded number for the upper end). The flux is generally close to being isotropic in all panels (b), (c) and (d) structures, especially in the second main one here, which has a large number density of $n \approx 2 \mathrm{~cm}^{-3}$ and a local beta value of $\beta \approx 0.2$. The less intense one just before does have more protons moving away from Earth, at $>90^{\circ}$ in the Northern Hemisphere, suggesting perhaps an Earth origin, but because it extends to $0^{\circ}$, it more likely has mirrored and straggling protons from a transient earthward flow burst (with almost no $\mathrm{O}^{+}$present). The last structure has some initially counter streaming protons at the highest energies (and maximum $\beta \approx 0.2$ ). It may be noted that the atmospheric loss cone at this large a distance, $\alpha \leq 1^{\circ}$, is very small compared to the
Table 1. Cluster satellite separations in GSE.

\begin{tabular}{|c|c|c|c|}
\hline \multicolumn{4}{|c|}{27 August 2002 at 18:00 UT } \\
\hline $\mathrm{SC}$ & $\Delta x, \mathrm{~km}$ & $\Delta y, \mathrm{~km}$ & $\Delta z, \mathrm{~km}$ \\
\hline $4-1$ & -4137 & 877 & -3081 \\
\hline \multicolumn{4}{|c|}{27 August 2002 at 21:30 UT } \\
\hline $\mathrm{SC}$ & $\Delta x, \mathrm{~km}$ & $\Delta y, \mathrm{~km}$ & $\Delta z, \mathrm{~km}$ \\
\hline $4-3$ & -2540 & -2304 & 715 \\
\hline $4-1$ & -3790 & 641 & -2452 \\
\hline $3-1$ & -1250 & 2945 & -3167 \\
\hline \multicolumn{4}{|c|}{28 August 2002 at 10:00 UT } \\
\hline $\mathrm{SC}$ & $\Delta x, \mathrm{~km}$ & $\Delta y, \mathrm{~km}$ & $\Delta z, \mathrm{~km}$ \\
\hline $4-1$ & -4108 & -1132 & 1367 \\
\hline \multicolumn{4}{|c|}{1 September 2003 at 02:00 UT } \\
\hline $\mathrm{SC}$ & $\Delta x, \mathrm{~km}$ & $\Delta y, \mathrm{~km}$ & $\Delta z, \mathrm{~km}$ \\
\hline $4-3$ & 66 & -102 & -242 \\
\hline $4-1$ & -21 & 82 & -199 \\
\hline $3-1$ & -87 & 184 & 43 \\
\hline
\end{tabular}

instrument angular resolution, so is not expected to noticeably affect the measured flux even when empty.

The bottom panel, (f), shows the GSE $z$ component of the tail magnetic field measured at SC1. It and the $y$ component are both small compared to $B_{x} \approx 37-40 \mathrm{nT}$ here, but it has an important recurring trend: it turns toward the northward direction in association with the plasma structures (the narrow wiggles on the far right are probably caused by a thin fieldaligned current moving past along the x-y plane).

The northward turning, or dipolarization, of the $B_{z}$ component is common to virtually all Cluster encounters with isolated high-latitude proton structures in the tail (hundreds examined). This effect is generally more pronounced the more energetic and/or denser the protons are, and often more pronounced than here, where the protons have rather soft energy spectrum. It is also associated with a diamagnetic weakening of the $B_{x}$ component, typically by several $\mathrm{nT}$ ( $B_{x}$ being stronger than $B_{z}$ to begin with), and a change in direction of the $B_{y}$ component, as well, although the turning of $B_{y}$ may be either duskward or, as in this case, dawnward (not shown).

Returning to panel (a) of Fig. 2, the seemingly energydispersed three-hour structure beginning about 17:45 UT on 27 August is in fact not a single dispersion, but a series of partially overlapping dispersions of varying duration and successively lower initial energies. The first few structures are shown on an expanded time scale in Fig. 3a, along with the simultaneous flux observed on SC1, in panel (b). The SC1 flux is also shown as function of pitch angle in panel (c), averaged over $1-40 \mathrm{keV}$, illustrating the typical initial arrival of earthward flux ( $<90^{\circ}$ here), followed by both earthward and mirrored flux. The several individual energy dispersions are partially obscured by spatial flux gradients, including sharp 


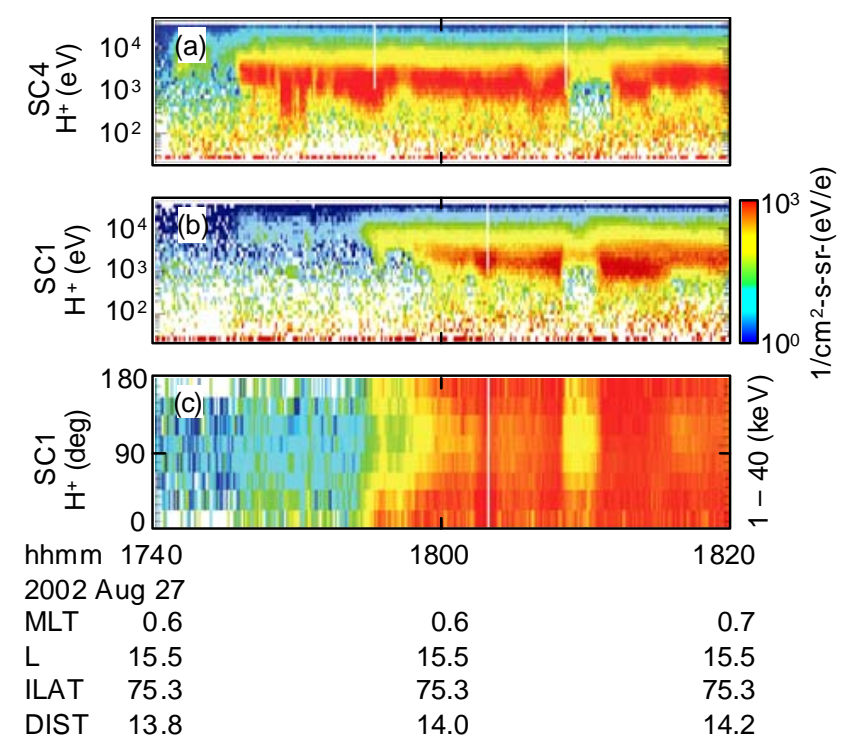

Fig. 3. (a) Expanded portion of Fig. 2a on 27 August. (b) Simultaneous proton flux at SC1. (c) Pitch-angle distribution of SC1 flux above $1 \mathrm{keV}$. Ephemeris is that of SC1 (see Table 1 for separations).

vertical edges. As shown in Table 1 (top), $\mathrm{SC} 1$ is earthward of SC4, but the distance in $x, 4137 \mathrm{~km}$, approximately parallel to the magnetic field, is much too short to explain the long delay ( $\sim 10 \mathrm{~min}$ ) of flux increase at $\mathrm{SC} 1$ relative to SC4. This "delay" is instead due to a transverse flux gradient, in $y$ or $z$.

The Fig. 3 structures do have energies, differential flux and peak number densities $\left(n \leq 1 \mathrm{~cm}^{-3}\right.$, not shown) that resemble those of a typical CPS, although not necessarily the local CPS subsequently encountered on this particular orbit (next section). According to first-order velocity moments (not shown), the earthward bulk flow speed of both panels (a) and (b) structures begins at about $300 \mathrm{~km} \mathrm{~s}^{-1}$ (single 8-s sampling at left edges) but declines to near zero within less than four minutes. Due to the large distance from Earth $\left(\sim 14 R_{E}\right.$ ), they have rather high beta values, $\beta$ reaching 0.47 at 18:16 UT in panel (a) (at local minimum $B \approx 44 \mathrm{nT}$ ), but subsequent structures within this same three-hour flux bundle have declining $\beta$ to $<0.1$ toward the end, despite decreasing $B$ due to the increasing distance from Earth. The (almost) two-hour bundle beginning near the end of 27 August in Fig. 2a actually has $\beta>1$ initially (for $\sim 20 \mathrm{~min}$ ), as well as larger initial energy. This one also has several substructures, some with clear downward energy dispersions (not shown).

\subsubsection{Equatorial $B_{x}$ reversal}

Of the multiple proton structures in the top panel of Fig. 2, only the one in the center contains reversals of the tail $B_{x}$ between northern and southern conditions. Except for the one just mentioned above (starting near midnight on $27 \mathrm{Au}-$ gust), this is also the only one that has beta values $\beta>1.0$. In fact, it contains several $B_{x}$ reversals and associated instances
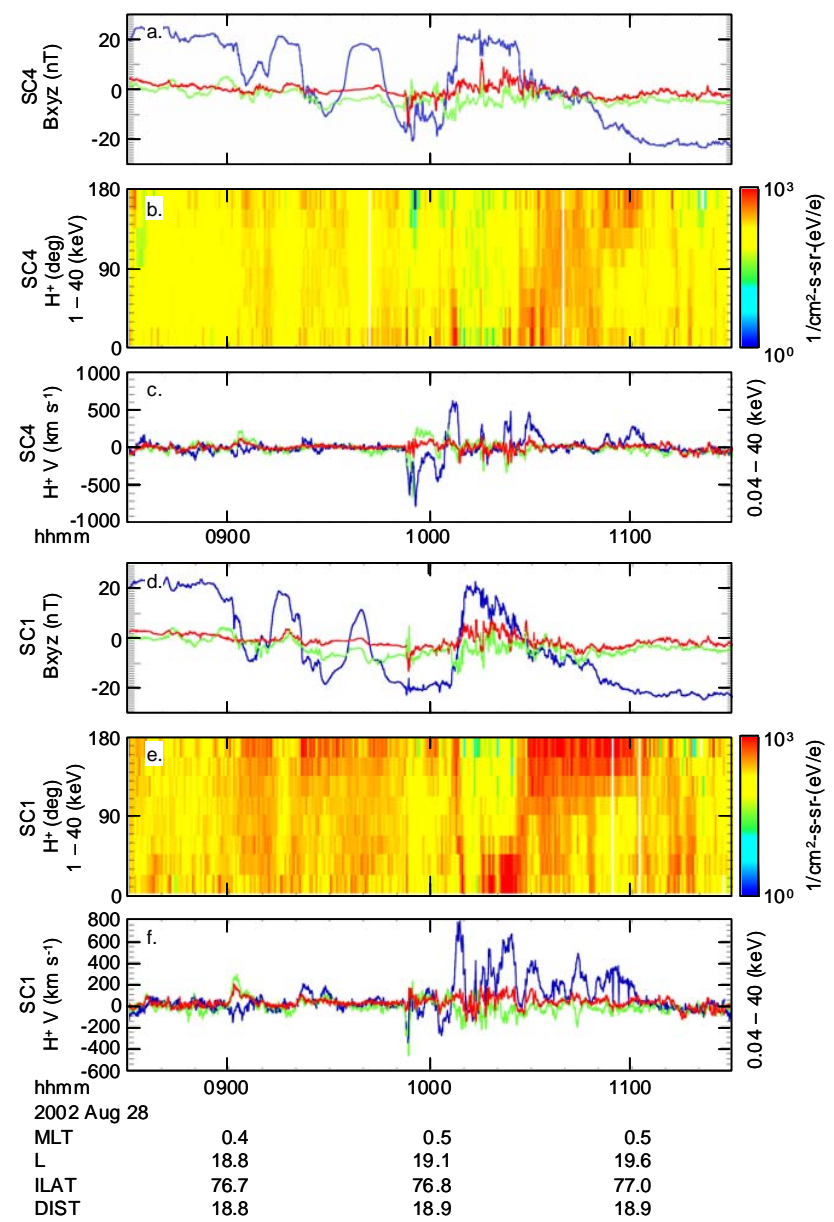

Fig. 4. (a) GSE magnetic field components $x$ (blue), $y$ (green), and $z$ (red), measured by SC4 near the apogee. (b) Corresponding proton flux pitch-angle distribution, averaged over 1-40 keV. (c) GSE proton bulk flow components $x$ (blue), $y$ (green), and $z$ (red), as inferred at SC4. (d-f) Same displays for SC1. Ephemeris at bottom is that of SC1 (cf. Table 1).

of $\beta \gg 1$, as seen by each of the southbound satellites over a two-hour interval between 09:00 and 11:00 UT on 28 August. These reversals are illustrated in panels (a) and (d) of Fig. 4 (blue lines) for SC4 and SC1, whose present separations are listed in Table 1 (28 August, 10:00 UT).

Note that the $\Delta y$ and $\Delta z$ separations are comparable to local $\mathrm{keV}$ proton gyroradii during these reversals, and yet the magnetic field differs significantly between the two locations, implying narrow proton pressure gradients as well. The average proton energy is about 5 to $8 \mathrm{keV}$ during these reversals (not shown), and the nominal gyroradius at $90^{\circ}$ pitch angle of a $5 \mathrm{keV}$ proton is $1018 \mathrm{~km}$ in a $10 \mathrm{nT}$ field, for instance. Therefore, both of the SC1 and SC4 CODIF instruments repeatedly sample protons with orbits that must deviate significantly from cycloidal here, at least at the higher energies, precluding strict adherence to a common $\boldsymbol{E} \times \boldsymbol{B}$ drift (cf. Chen et al., 2000). This may be fairly normal conditions 
about the midplane this far from Earth. The three-hour interval ending at 09:00 UT has $K_{p}=2$, and the following two three-hour intervals both have $K_{p}=1+$.

Observing multiple $B_{x}$ reversals over a one- to three-hour time span is typical of the near-apogee Cluster crossings of the nominal equatorial plane, although the measured magnetic field is often more complex than here. This is consistent with some degree of latitudinal oscillation, or "flapping," of the plasma sheet, but that kind of bulk motion does not readily explain the satellites' encounters with more or less isolated proton structures at higher latitude, where $B_{x}$ retains its direction, $\left|B_{x}\right|$ is greater, and beta is less or much less than one. Most, if not all, of the higher-latitude structures may have formed by proton motion away from the equator and toward Earth, both northward and southward, along newly reconfigured and closed magnetic field lines. For a conceptual illustration of this scenario, see Fig. A1 in the Appendix.

The pitch-angle spectra (1-40 keV) in panels (b) and (e) of Fig. 4 in fact show direct evidence of proton flows away from the midplane, especially the $\mathrm{SC} 1$ spectrum in panel (e). That is, where flux is enhanced (red color), it mostly occurs at pitch angles in the earthward direction, whether $B_{x}$ is negative (flows at $>90^{\circ}$ ) or positive (flows at $<90^{\circ}$ ). This earthward flux is also reflected in the calculated first-order velocity moments in panels (c) and (f) (different scales), where $v_{x}$ (blue lines) is preferentially positive, especially in panel (f), although narrow density gradients (not shown) in combination with large proton gyroradii may have some spurious effects.

It is important to note here that the earthward proton flow bursts are more numerous and stronger at SC1 (panels e and f) than they are at SC4 (panels b and c), in spite of the fact that $\mathrm{SC} 1$ is about $4000 \mathrm{~km}$ closer to Earth, and the $y$ and $z$ separations are only about $1000 \mathrm{~km}$ each (Table 1 ). This implies that the near-equatorial plasma density between the two satellites may be undergoing depletion, but the zero-order velocity moments in this case (not shown) are inconclusive in that regard. If there is a trend toward the end of this time interval, the satellites may be out of reach, having moved too far from the midplane. There are, however, numerous equatorial crossings in the Cluster data that are consistent with local and transient plasma sheet depletion. One good example from almost the same identical geomagnetic location as Fig. 4, one year later, follows below (see also the recent Cluster results of Asano et al., 2008).

\subsection{September 2003}

Early on this day, $B_{x}$ at the Cluster satellites remains between $\pm 10 \mathrm{nT}$ for almost four hours while undergoing numerous reversals. Figure 5 shows a 20 -min subinterval with several transient and presumably localized proton density depletions in the tail midplane (panels a, c and d), the most dramatic one occurring within a single 8-s sampling at about 01:56:30 UT. Only SC1 data are shown here, since the sep-
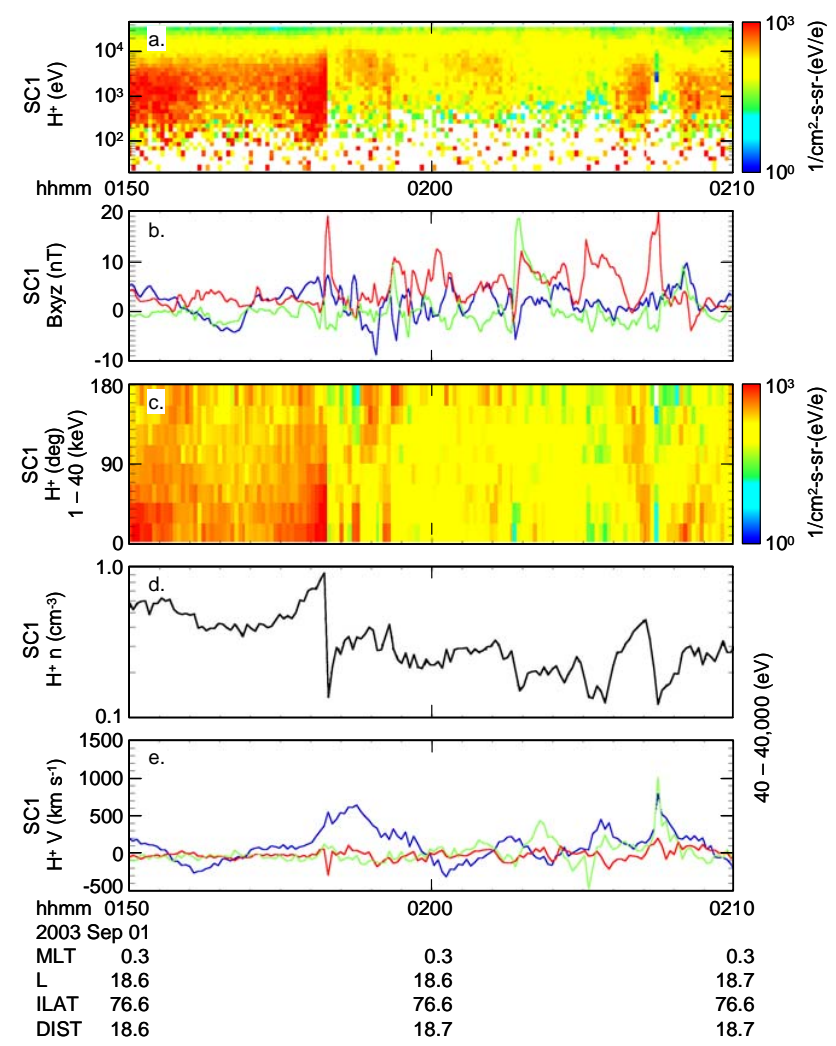

Fig. 5. 20-min interval of SC1 observations near the apogee of a different orbit (in 2003). (a) Flux versus time and energy. (b) GSE magnetic field components $x$ (blue), $y$ (green), and $z$ (red). (c) Flux versus time and pitch angle (1-40 keV average). (d) Proton density. (e) GSE proton bulk flow components $x$ (blue), $y$ (green), and $z$ (red) (cf. Table 1).

arations at this time, listed in Table 1 (bottom), are smaller than local $\mathrm{keV}$ proton gyroradii, and the three sets of data are nearly identical (as expected). For example, the three sets of spin-averaged magnetic field components agree to within about $\pm 1 \mathrm{nT}$.

It is important to note here that all major downward slopes in the panel (d) density profile are closely associated with enhanced northward $B_{z}$ in panel (b) (red line), often to the extent that $B_{z}$ becomes the dominant component. One of these density drops, at about 02:03 UT, also involves enhanced (duskward) $B_{y}$ (green line).

The bottom panel of Fig. 5 shows that at least three of the density drops in panel (d) are also associated with an increased earthward bulk $v_{x}$ (blue line), the last of which also has a large spike in $v_{y}$ (green line), as calculated from the measured differential flux. As with Fig. 4, proton density gradients may have some spurious effects, but the fact that the three largest $v_{x}$ values are all positive is reassuring, being that past statistical studies of high-speed ion flows in the plasma sheet showed them strongly peaked in the sunward direction (e.g. Baumjohann et al., 1990). 


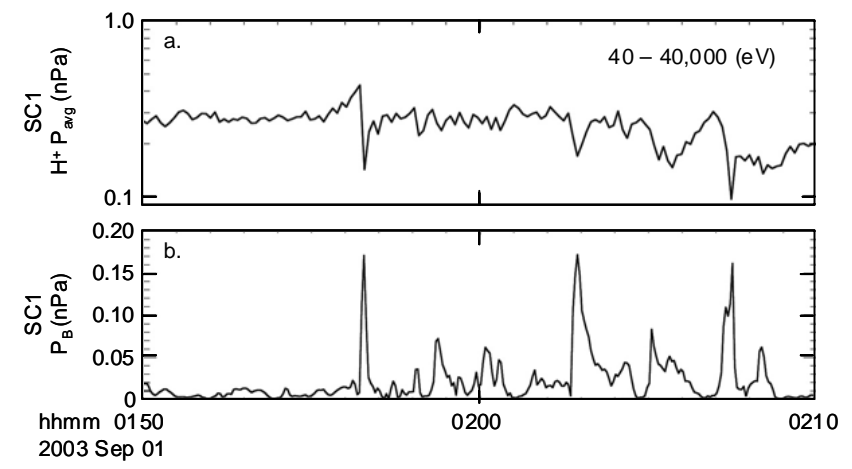

Fig. 6. (a) Proton pressure during the Fig. 5 time interval. (b) Simultaneous magnetic pressure.

According to panels (b), (c) and (e), the initial growth of the first positive peak in $v_{x}$ includes earthward fieldaligned proton flow ( $B_{x}$ being earthward) associated with the pileup of density in panel (d), which is followed at about 01:56:30 UT by a precipitous drop in density coincident with the sudden spike in $B_{z}$. The $v_{x}$ continues to grow for another minute, peaking at $600 \mathrm{~km} \mathrm{~s}^{-1}$, and it remains positive until about 02:00 UT $\left(\int v_{x} d t \sim 13 R_{E}\right)$. This suggests that the first large $B_{z}$, at least, is associated with a rapidly contracting closed magnetic loop. If one assumes that the local magnetic field in this case does move with the plasma, then what appears as a mere 8 -s spike in $B_{z}$ in the s/c frame of reference may have a true extent of almost $1 R_{E}$ in $x$. Much the same can be said about the last positive peak in $v_{x}$, as well, although it also involves large duskward $v_{y}$ (green line).

It may be noted that the local CPS proton population in this event, prior to the sudden drop in flux and density, is more similar to the Fig. 3 population than was the CPS population described above. The associated proton and magnetic field pressures are illustrated in Fig. 6 (note the different panels a and b scales). By comparison with panel (d) in Fig. 5, the proton pressure is seen to have somewhat smaller relative variations over time than does the proton number density, mainly because it is less dependent on lower-energy protons.

There is little going on in the way of global magnetic activity at this time. The first three hours of 1 September, 00:0003:00 UT, have $K_{p}=2$, and the preceding last three hours of 31 August had $K_{p}=1$. It is only during the following six hours of 1 September, 03:00-09:00 UT, that activity is moderately enhanced to $K_{p}=4-$ and $3+$.

\section{Discussion}

Even without the benefit of having co-travelers, each of the Cluster satellites affords a unique perspective of the plasma sheet from its very large near-polar orbit. Considering SC4 in the top panel of Fig. 2, one is challenged to describe what is meant by a plasma sheet "boundary layer" (PSBL), as op- posed to a "central" plasma sheet, concepts that were developed from data taken in "near-equatorial" orbits, especially with the ISEE-1 and ISEE-2 satellites (with apogees near $23 R_{E}$; see e.g. Parks et al., 1984; Eastman et al., 1985). Closely related are the concepts of plasma sheet "thinning," "dropout" and "recovery," as also inferred from nearequatorial observations (e.g. Hones et al., 1973, 1986; Lui et al., 1975). The beginning of dense (red) flux in Fig. 3a would qualify as plasma sheet recovery at SC4 according to the ISEE standards (e.g. Lennartsson, 1997). The fact that a somewhat similar flux increase is seen about 10 min later at SC1 might even seem consistent with northward expansion of the plasma sheet, being that SC1 is further north (Table 1). However, the bulk motion derived from velocity moments has fluctuating $y$ and $z$ components at both satellites, and the average $v_{z}$ is weakly southward at SC4 at the time $(\sim 10-$ $20 \mathrm{~km} \mathrm{~s}^{-1}$, not shown) and near zero at SC1. Actually, faint (light blue) flux appears at SC1 about the same time (and same energies) as the dense flux at $\mathrm{SC} 4$, indicating proton arrival along the magnetic field ( $\sim x$-directed), and it remains faint for almost $10 \mathrm{~min}$ due to a cross-field density gradient, probably north-south directed $\left(\Delta z \approx 0.5 R_{E}\right)$.

In terms of earthward field-aligned flow, the structure beginning about 17:53 UT in Fig. 3a, for instance, has fairly continuous downward dispersion on the bottom, whose slope suggests a source about $20 R_{E}$ tailward of SC4, or at GSE $x \approx-30 R_{E}$. The subsequent adjacent structures in this flux bundle (Fig. 2a), especially those after 19:00 UT, are less energetic than typical proton bursts (Lennartsson et al., 2001). The end of the bundle consists of a fairly steep downward slope (from $\sim 4 \mathrm{keV}$ to $\sim 0.1 \mathrm{keV}$ in five minutes). $K_{p}$ is 2 between 18:00 and 21:00 UT, having been 3- before and becoming 3+ afterward.

The trailing narrow structures shown with expanded time scale (one hour) in panel (b) of Fig. 2 are still less energetic and have unusually large flux at near-zero energy. These are not necessarily representative of bursty flux, but they do highlight the inherent spatial fine structure of highlatitude proton flux and allow for fairly simple multipoint inter-comparison. The second structure in panel (b), for example, may seem to be the same as the first one in panel (c) and the second one in panel (d). If those are the same one, then it either has some kind of curved shape across the magnetic field, to appear in the observed succession at the three satellites, given the separations in Table 1, or, if planar, is drifting eastward and southward. The true situation is probably some combination of shape and drift. For comparison, Fig. 7 shows SC1 velocity moments for the first two structures in Fig. 2d.

The main (second) structure has a moment-based GSE $v_{y}$ (panel d) that tends westward, but its $v_{z}$ (bottom) is indeed southward, at some $20-50 \mathrm{~km} \mathrm{~s}^{-1}$, after the initial large northward peak. The corresponding $v_{y}$ and $v_{z}$ of the second structure in Fig. $2 \mathrm{~b}$ are very similar, including the large but brief opposite peaks (not shown), while delayed by about 
$1.5 \mathrm{~min}$. This delay is fairly consistent with those $v_{y}$ and $v_{z}$ moments, but $\mathrm{SC} 3$ is further west and south than SC4, and yet the first peak in Fig. 2c is slightly ahead of the SC1 observation (by about $0.5 \mathrm{~min}$ ). It may be a separate structure, or its shape is curved (convex north-westward).

The brief velocity peaks $\left(\sim 150 \mathrm{~km} \mathrm{~s}^{-1}\right)$ in panels (d) and (e) of Fig. 7 demonstrate the kind of effects of steep proton density gradients that was alluded to in the previous sections. The negative (dawnward) $v_{y}$ peak in panel (d), along with the corresponding mean energy in panel (b), is consistent with the initial brief encounter by SC1 of only the most energetic gyrating protons (those with the largest gyroradii) on the bottom side of a structure that is drifting southward. The positive (northward) $v_{z}$ peak in panel (e), eight seconds later (next sampling), has a less straightforward interpretation but may reflect a curved or sloping edge. Similar paired peaks $\left(<100 \mathrm{~km} \mathrm{~s}^{-1}\right)$ are observed by SC4 1.5 min later, within one single 8-s sampling (not shown). As evident from panel (b) of Fig. 2, the left (leading) edge of this (second) structure indeed appears within a single 8-s sampling at energies below a couple of keV. Even at $50 \mathrm{~km} \mathrm{~s}^{-1}$ drift speed, this means a density gradient scale length less than three times the gyroradius of a $2 \mathrm{keV}$ proton (at $90^{\circ}$ pitch angle).

Panels (b), (c) and (d) of Fig. 2 together virtually prove that there are isolated plasma filaments, as opposed to mere folds in a single continuous boundary layer. The isotropic pitchangle distribution within three of the filaments in panel (e), and the absence of energy dispersion in panel (d), may mean that the filaments in this case extend between the hemispheres and have reached near-uniform density. The satellites are within a generally tail-like magnetic field, but the field becomes less stretched in association with the filaments themselves, according to panel (f), especially with the second one in panels (d) and (e). Given the locally equatorward (southward) bulk motion of this same structure in Fig. 7e, it may very well be a magnetically closed and contracting plasma loop, similar to the modeled "mature bubble" structure in Figs. 3 and 4 of Chen and Wolf (1993).

Having a plasma filament form that connects high-latitude points on opposite sides of the main plasma sheet requires a localized reduction of the midplane (maximum) plasma pressure. That this scenario is feasible follows from Fig. 4, where earthward bulk plasma flow is at times stronger at $\mathrm{SC} 1$ than at $\mathrm{SC} 4$, even though SC1 is $\sim 4000 \mathrm{~km}$ closer to Earth. Growing local magnetic field strength $B$ counteracts the plasma pressure reduction by betatron acceleration, but only in proportion to $B$, whereas the magnetic pressure (and tension) grows in proportion to $B^{2}$. Once higher-latitude magnetic field lines do connect across the tail midplane, the plasma there is able to expand rapidly to higher latitude as well, leading to further diamagnetic imbalance. The logical consequence is earthward contraction of the flux tube, in accordance with the "plasma bubble" (buoyancy force) theory of Pontius and Wolf (1990) and Chen and Wolf (1993) (see also Birn et al., 2004).
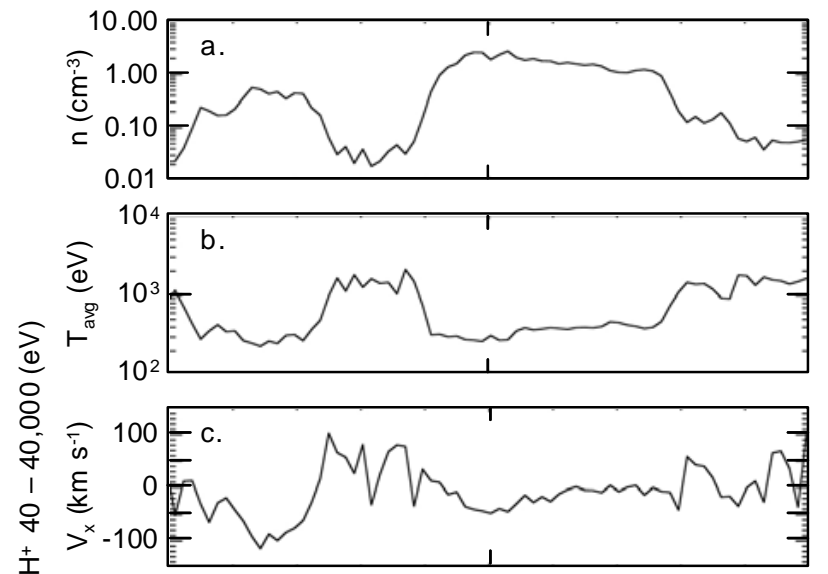

is
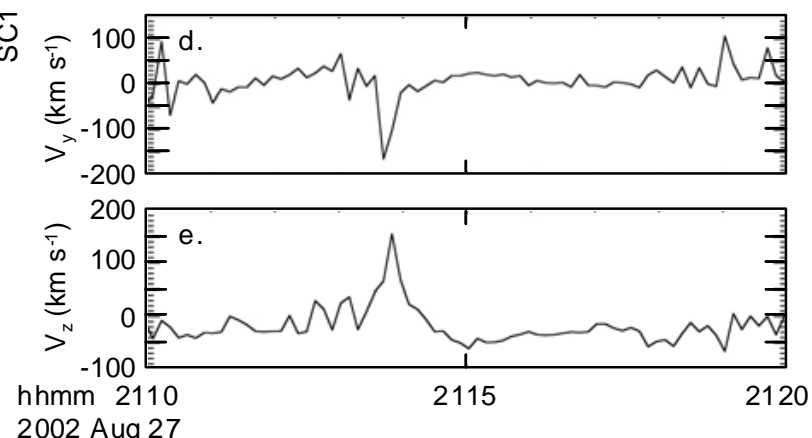

2002 Aug 27

Fig. 7. Velocity moments $(40-40000 \mathrm{eV})$ of the first two flux structures in Fig. 2d: (a) density, (b) thermal energy, and (c-e) bulk velocity components $v_{x}, v_{y}$, and $v_{z}$, respectively.

Figure 5 bears out this scenario with multiple dipolarizations and associated plasma density drops. Most of this time interval fits the definition of a "typical" 10-min BBF event (e.g. Figs. 3 and 5 in Angelopoulos et al., 1992), but the first and largest density drop, at 01:56:30 UT, arguably occurs during the most "archetypical" single BBF flow burst. In fact, the associated time series of magnetic field and proton density and bulk flow have nearly identical shapes as those in Fig. 4 of Ohtani et al. (2004), which show superposed epoch analysis of 818 fast earthward flow events that occurred at $-31<\mathrm{GSM} x<-5 R_{E}$. The main differences are smaller amplitudes in the superposed case. It may be noted that the first large spike in $B_{z}$ in Fig. 5 above $(19 \mathrm{nT}$, red line) is four times larger than the local Earth vacuum dipole field, and causes the proton beta to briefly fall below one $(\beta \approx 0.7)$. Similar low-beta conditions are reached with the subsequent large spikes in $B_{y}(19 \mathrm{nT}$, green, $\beta \approx 0.9)$ and $B_{z}$ ( $20 \mathrm{nT}, \beta \approx 0.6$ ). This is illustrated in Fig. 6 . Protons are the dominant ionic component at this time (virtually no $\mathrm{O}^{+}$ ions present), so Fig. 6 can thus be said to justify the original assumptions by Pontius and Wolf (1990) about "plasma bubbles" having both lower particle pressure and stronger magnetic field than their surroundings in the tail midplane. 
The recurring but short-lived (in the s/c frame) enhancements of $B_{z}$ at low latitude, combined with the associated local density drops, are quite consistent with the appearances of multiple, transient and/or localized proton structures at higher latitude, along with some northward turning $B_{z}$ there as well. There is in fact a brief southward $v_{z}$ in panel (e) of Fig. 5 (red line) coincident with the first $B_{z}$ spike in panel (b), and a northward $v_{z}$ with the last $B_{z}$ spike, showing fieldaligned flows with some degree of north-south asymmetry at those times. The flux structures in Fig. 2d are not typical of high-latitude proton bursts in progress, but the behavior of $B_{z}$ in Fig. $2 \mathrm{f}$ is representative of isolated flux structures with energy and pitch-angle dispersion as well.

The very large peak values of $B_{z}$ in Fig. 5b, 19-20 nT, are indeed consistent with flux tubes that touch the lobes nearby, as illustrated by the dashed curve in Fig. A1 in the Appendix. This is because the sum of kinetic and magnetic pressures in Fig. 6 remains mostly at or below $0.3 \mathrm{nPa}$, implying that the adjacent lobe $\left|B_{x}\right| \leq 27 \mathrm{nT}$ at pressure balance, i.e. a mere $30-40 \%$ larger. Where the flux tubes bend earthward, away from the equatorial plane, they can reasonably be expected to narrow and have a field that quickly reaches that lobe strength. This is consistent with prior equatorial (and local) "diamagnetic motion" of the lobe field (see Appendix) and differs somewhat from the MHD model of Birn et al. (2004), where $B_{x}$ is constrained by the boundary conditions (their Fig. 5), and the equatorial $B_{z}$ remains about an order of magnitude weaker than the adjacent lobe field during the simulated earthward flux tube contraction (their Figs. 4 and 9).

The recurring earthward proton flows in Fig. 5, with their embedded positive $B_{z}$ peaks, imply that (some) plasma located tailward of Cluster remains magnetically connected to Earth. This raises the issue of whether BBFs may form without generating magnetic loops with negative $B_{z}$ travelling in the tailward direction, away from a magnetic neutral line (or point). Tail plasma flows with negative $v_{x}$ and $B_{z}$ are certainly observed part of the time (e.g. Ohtani et al., 2004; Asano et al., 2008), and they are generated in the Birn et al. MHD model. Assuming that each earthward BBF flow burst is initially formed together with a tailward-flowing counterpart, then Fig. 5 shows that each burst only empties some rather limited portion of the downtail plasma sheet, for instance some very narrow longitudinal sector.

Even while depicting only local conditions, Figs. 5 and 6 show two important aspects of "tail current disruption" (e.g. Lui et al., 2007). For one, sufficient proton density is a crucial condition for the plasma sheet's diamagnetic strength, and its diamagnetism plays a crucial role in keeping the two tail lobes' $B_{x}$ fields apart, i.e. keeping pressure balance. For another, a strongly enhanced $B_{z}$ component in the midplane acts as a barrier against a net dawn-to-dusk proton drift current. The scenario advocated here assumes that some initial local reduction in the midplane $\beta$ causes higher-latitude $B_{x}$ to be diverted into midplane $B_{z}$, causing further reduction of both particle pressure and $\beta$. This may be envisioned to develop rapidly, thereby launching field-aligned proton bursts, northward and southward, as well as an earthward BBF (plasma bubble).

\section{Summary}

The high-latitude "boundaries" of the plasma sheet, at least on the nightside, are dynamic latitude zones of recurring and transient (minutes to tens of minutes) earthward and magnetic field-aligned bursts of plasma, whose ionic component is dominated by protons with flux, energies, and densities that are consistent with a central plasma sheet source at varying distance, the latter often indicated by downward dispersion of thermal energy at varying rates. The source can be expected to be as close as the $\sim 19 R_{E}$ Cluster apogees at times, or closer still (e.g. Lennartsson et al., 2001; Sauvaud and Kovrazhkin, 2004).

These high-latitude proton bursts, collectively known as the PSBL, have time scales that compare with those of the central plasma sheet (CPS) BBFs, i.e. bulk flow events with large, positive and bursty GSE $v_{x}$, although the statistics obtained for the BBFs have been limited to GSE $x>-31 R_{E}$ (e.g. Baumjohann et al., 1990; Angelopoulos et al., 1992; Petrukovich et al., 2001; Raj et al., 2002; Ohtani et al., 2004; Cao et al., 2006). What makes the known BBFs plausible sources for the PSBL bursts are, to begin with, (1) their earthward-directed bulk motion and (2) their association with a transient and large increase of the local tail $B_{z}$ component (local dipolarization). The enhanced $B_{z}$ provides intermittent access to higher latitudes for the CPS plasma, resulting in transient and local CPS density reductions, as illustrated by Fig. 5 above and implied by the extensive epoch analysis of Ohtani et al. (2004). Their earthward sense of bulk motion ("flux tube contraction") is consistent with the lack of distinctive latitudinal dispersion of the PSBL burst energies (Lennartsson et al., 2001).

Another sign of kinship between the PSBL bursts and the BBFs is their similar spatial fine structure. The PSBL bursts have prominent filaments aligned along the magnetic field with transverse flux gradients that are often characterized by $\sim 10 \mathrm{keV}$ proton gyroradii scale size (Lennartsson et al., 2007) or even smaller (recall discussion of the second structure in Fig. $2 \mathrm{~b}$ above). The same kind of fine structure is also found during Cluster near-apogee traversals of the tail midplane, as illustrated above by the Fig. 4 differences between SC1 and SC4 magnetic field strengths (anti-correlated with proton pressures) and bulk flow velocities, given the rather small $\Delta y$ and $\Delta z$ separations in Table 1 . The $10-\mathrm{keV}$ type proton gyroradii scale size apparently applies to BBFs in general, according to the different BBF statistics obtained by Cao et al. (2006) between 2001 and 2002 (see second last paragraph of the above Introduction). 
A third and indirect connection are the observations by e.g. Angelopoulos et al. (1997) and Sergeev et al. (2000a, b) of a good correlation between BBFs in the tail and ionospheric auroral activity, even when ground magnetic signatures are absent. This ties in with the spatial fine structure of both the BBFs and the PSBL proton bursts, since proton gyroradiiscale burst density gradients are likely to generate electrostatic shocks close to Earth (Lennartsson, 2003) and thus in turn drive electron precipitation (Lennartsson, 2006).

A fourth and theoretical kind of connection is offered by the "plasma bubble" (buoyancy force) theory of Pontius and Wolf (1990) and Chen and Wolf (1993). The Cluster proton and magnetic field data examined here have several aspects that mesh well with this theory. In particular, Figs. 5 and 6 show BBF-type earthward proton flow bursts to have both lower particle pressure and stronger magnetic field than their surrounding equatorial plasma. This combination yields the magnetic buoyancy force that propels the theoretical plasma bubbles earthward at speeds in the range of reported BBF speeds. According to the Chen and Wolf analysis, each moving plasma bubble occupies a magnetic flux tube that is more dipole-shaped than surrounding flux tubes and therefore reaches higher latitudes than those in the earthward direction. Once filled uniformly with plasma, the bubble's flux tube, according to Chen and Wolf, will have higher particle pressure than its surroundings along most of its length, away from the equatorial plane. This accounts for more or less isolated high-latitude plasma filaments (Figs. 2, 3 and 7), whose formation may be triggered by local reductions of the equatorial $\beta$ (Fig. 4).

\section{Appendix A}

\section{Magnetic field motion}

As one traces a tail magnetic field line toward Earth, be it from $P_{1}$ to $F_{1}$ in Fig. A1, or from $P_{2}$ to $F_{2}$, et cetera, the local field is known to approach Earth's internally generated and approximately dipole-shaped field in a smooth fashion, without any sharp bends in direction below a few $R_{E}$ altitude (e.g. Tsyganenko, 1990). Nonetheless, it is commonly postulated that the field lines through $P_{1}$ and $P_{1}^{\prime}$, while each being connected to the solar wind at greater distance, are continually "moving" tailward and equatorward at the $\boldsymbol{E} \times \boldsymbol{B} \cdot \boldsymbol{B}^{-2}$ velocity, along with ions and electrons, $\boldsymbol{E}$ being directed duskward, via positions like those of the field lines through $P_{2}$ and $P_{2}^{\prime}$. At some distance down the tail, perhaps anywhere between 100-200 $R_{E}$ or so, the oppositely directed Northern and Southern Hemisphere field lines, are believed to come together and form an extended equatorial cross-tail "neutral line" ("X-line"), where the field-line (and plasma) motion is diverted and divided into both tailward and earthward components, the latter one sustaining the plasma sheet.

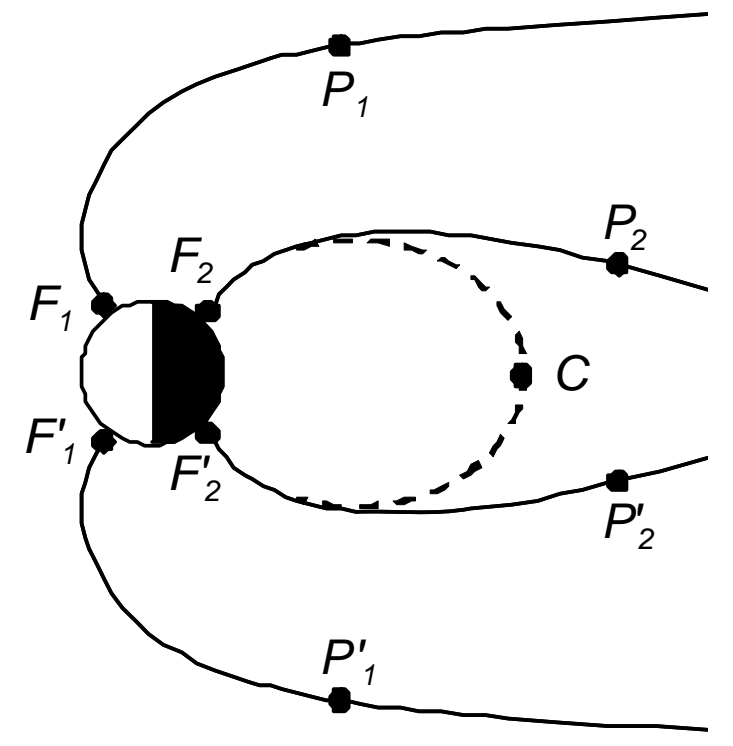

Fig. A1. Schematic tail lobe magnetic field lines near noonmidnight plane (solid lines; not to scale) and a "reconnected" field line (dashed; tailward moving counterpart not drawn). Letters $F$ and $F^{\prime}$ denote field line intersection with Earth's surface (see text).

This lobe magnetic field-line motion is thought to be a necessary consequence of large electric conductance along the field lines in the upper ionosphere and beyond, leading to the "frozen-in condition," whereby the magnetic flux through any closed contour attached to the moving plasma remains constant. (The ideal formula is derived by Alfvén and Fälthammar (1963, Sect. 3.9), but the authors caution against idle use of this condition.) The "large field-aligned conductance" may or may not be a good assumption, considering that (1) the measured lobe plasma density earthward of a few tens of $R_{E}$ is mostly low and (2) the charge carriers are subject to magnetic mirroring. In any case, it does not apply to the near-Earth field within the neutral atmosphere.

The $F$ and $F^{\prime}$ foot points are electrostatically insulated from the external magnetic field, and the local field strength is mainly (>99\%) from Earth's dipole and must follow Earth's rotation along constant geographic latitude. This differs greatly from the observed horizontal circulation of ionosphere plasma (e.g. Ruohoniemi and Baker, 1998), so if the ionosphere $\boldsymbol{E} \times \boldsymbol{B}$ drift were to physically carry with it the magnetic field, then there would have to be sharp bends in the near-Earth field lines, being that $\boldsymbol{B}$ is divergence free, and these bends are not observed.

On a finer spatial scale, auroral arcs are believed to align beneath electric potential structures with a V-shaped cross section, whose transverse electric field $\boldsymbol{E}_{\perp}$ is indeed observed above a few thousand $\mathrm{km}$ altitude by polar orbiting satellites, as paired and opposite vectors (e.g. Mozer et al., 1977). The large (and growing) shear that the magnetic field would display, if it were to move with the associated opposite $\boldsymbol{E} \times \boldsymbol{B}$ 
drifts, is not observed, however, only a small shear caused by the field-aligned sheet current carried by the precipitating electrons. In other words, there are prevalent and verifiable circumstances under which particle $\boldsymbol{E} \times \boldsymbol{B}$ drift does not entail magnetic field motion. Furthermore, the magnetic field frozen-in condition, in the sense of constant flux through any closed contour moving with the plasma, is contrary to diamagnetism, when plasma compressibility is taken into account, since it requires that the magnetic field strength (and pressure) increase with increasing plasma density (and pressure).

On the other hand, the diamagnetic property of the plasma can cause the magnetic field to move in a transient fashion, either by being displaced by increasing plasma pressure or by filling in where plasma pressure is decreasing. With reference to Fig. A1, the stretched shape of the solid field lines represents the end response to external solar wind and internal plasma sheet pressures (allowing for diamagnetic pressure balance; e.g. Fairfield et al., 1981). The plasma sheet may well fill from the tail flanks (e.g. Eastman et al., 1976; Orsini et al., 1990), specifically via inward $\boldsymbol{E} \times \boldsymbol{B}$ drift in the low latitude boundary layer (LLBL) fringe electric field (as required by $\operatorname{curl} \boldsymbol{E}=0$; see Lennartsson, 1992, 1997), while "pushing" the lobe magnetic field into its tail-like shape. In fact, the entry of solar wind plasma into Earth's plasma sheet seems to have, by itself, a stabilizing effect on the magnetotail, since the near-Earth plasma sheet has the most numerous and least energetic $(\sim 1 \mathrm{keV}$ per nucleon) protons and alpha particles during extended periods (many hours) of extreme geomagnetic quiescence (e.g. Lennartsson, 1992, and references therein).

If one considers, for the sake of argument, that the plasma pressure at point $C$ in Fig. A1 is suddenly reduced below diamagnetic equilibrium, then one can imagine that the lobe magnetic field moves locally to penetrate the midplane (reconnect), as indicated by the dashed line. This in turn will allow plasma remaining at point $C$ to expand to higher latitude, and earthward, further reducing its midplane pressure, probably causing the magnetic loop to contract earthward. The latter can be envisioned with the "bubble picture" of Chen and Wolf (1993), where the newly closed flux tube (bubble) is propelled earthward by a magnetic buoyancy force related to the interchange instability (see also Birn et al., 2004). Sudden closures of high-latitude flux tubes through the midplane would realize one of Williams (1981) proposed sources of energetic ion beams observed with ISEE 1 at the edge of the plasma sheet, namely "random encounters of the ISEE 1 flux tube with a steady source." This is essentially the process elaborated on here with the help of Cluster data.

Acknowledgements. O. W. Lennartsson thanks the Cluster CIS Teams at UC Berkeley and UNH, Durham, for making their data analysis software available, and K. J. Trattner at the Lockheed Martin ATC for many helpful discussions. $K_{p}$ and other ground magnetic measures were obtained from the Kyoto University Web site for geomagnetic indices. This work was supported by NASA un- der grants NAG5-13211 and NNX07AQ88G and contract NAS530302.

Topical Editor I. A. Daglis thanks one anonymous referee for her/his help in evaluating this paper.

\section{References}

Alfvén, H. and Fälthammar, C.-G.: Cosmical Electrodynamics, Fundamental Principles, Clarendon Press, Oxford, 1963.

Angelopoulos, V., Baumjohann, W., Kennel, C. F., Coroniti, F. V., Kivelson, M. G., Pellat, R., Walker, R. J., Lühr, H., and Paschmann, G.: Bursty bulk flows in the inner central plasma sheet, J. Geophys. Res., 97, 4027-4039, 1992.

Angelopoulos, V., Kennel, C. F., Coroniti, F. V., et al.: Statistical characteristics of bursty bulk flow events, J. Geophys. Res., 99, 21257-21280, 1994.

Angelopoulos, V., Phan, T. D., Larson, D. E., et al.: Magnetotail flow bursts: association to global magnetospheric circulation, relationship to ionospheric activity and direct evidence for localization, Geophys. Res. Lett., 24, 2271-2274, 1997.

Asano, Y., Nakamura, R., Shinohara, I., et al.: Electron flat-top distributions around the magnetic reconnection region, J. Geophys. Res., 113, A01207, doi:10.1029/2007JA012461, 2008.

Balogh, A., Carr, C. M., Acuña, M. H., et al.: The Cluster Magnetic Field Investigation: overview of in-flight performance and initial results, Ann. Geophys., 19, 1207-1217, 2001,

http://www.ann-geophys.net/19/1207/2001/.

Baumjohann, W., Paschmann, G., and Lühr, H.: Characteristics of high-speed ion flows in the plasma sheet, J. Geophys. Res., 95, 3801-3809, 1990.

Birn, J., Raeder, J., Wang, Y. L., Wolf, R. A., and Hesse, M.: On the propagation of bubbles in the geomagnetic tail, Ann. Geophys., 22, 1773-1786, 2004,

http://www.ann-geophys.net/22/1773/2004/.

Bosqued, J. M., Ashour-Abdalla, M., El Alaoui, M., Zelenyi, L. M., and Berthelier, A.: AUREOL-3 observations of new boundaries in the auroral ion precipitation, Geophys. Res. Lett., 20, 12031206, 1993.

Cao, J. B., Ma, Y. D., Parks, G., et al.: Joint observations by Cluster satellites of bursty bulk flows in the magnetotail, J. Geophys. Res., 111, A04206, doi:10.1029/2005JA011322, 2006.

Chen, C. X. and Wolf, R. A.: Interpretation of high-speed flows in the plasma sheet, J. Geophys. Res., 98, 21409-21419, 1993.

Chen, L.-J., Parks, G. K., McCarthy, M., Larson, D., and Lin, R. P.: Kinetic properties of bursty bulk flow events, Geophys. Res. Lett., 27, 1847-1850, 2000.

Eastman, T. E., Hones Jr., E. W., Bame, S. J., and Asbridge, J. R.: The magnetospheric boundary layer: Site of plasma, momentum and energy transfer from the magnetosheath into the magnetosphere, Geophys. Res. Lett., 3, 685-688, 1976.

Eastman, T. E., Frank, L. A., and Huang, C. Y.: The boundary layers as the primary transport regions of the Earth's magnetotail, J. Geophys. Res., 90, 9541-9560, 1985.

Fairfield, D. H., Lepping, R. P., Hones Jr., E. W., Bame, S. J., and Asbridge, J. R.: Simultaneous measurements of magnetotail dynamics by IMP spacecraft, J. Geophys. Res., 86, 1396-1414, 1981.

Forbes, T. G., Hones Jr., E. W., Bame, S. J., Asbridge, J. R., Paschmann, G., Sckopke, N., and Russell, C. T.: Evidence for 
the tailward retreat of a magnetic neutral line in the magnetotail during substorm recovery, Geophys. Res. Lett., 8, 261-264, 1981.

Grigorenko, E. E., Sauvaud, J.-A., and Zelenyi, L. M.: Spatialtemporal characteristics of ion beamlets in the plasma sheet boundary layer of magnetotail, J. Geophys. Res., 112, A05218, doi:10.1029/2006JA011986, 2007.

Hones Jr., E. W., Asbridge, J. R., Bame, S. J., and Singer, S.: Substorm variations of the magnetotail plasma sheet from $X_{S M} \sim-6 R_{E}$ to $X_{S M} \sim-60 R_{E}$, J. Geophys. Res., 78, 109$132,1973$.

Hones Jr., E. W., Fritz, T. A., Birn, J., Cooney, J., and Bame, S. J.: Detailed observations of the plasma sheet during a substorm on April 24, 1979, J. Geophys. Res., 91, 6845-6859, 1986.

Huang, C. Y., Frank, L. A., Peterson, W. K., Williams, D. J., Lennartsson, W., Mitchell, D. G., Elphic, R. C., and Russell, C. T.: Filamentary structures in the magnetotail lobes, J. Geophys. Res., 92, 2349-2363, 1987.

Keiling, A., Reme, H., Dandouras, I., et al.: Transient ion beamlet injections into spatially separated PSBL flux tubes observed by Cluster-CIS, Geophys. Res. Lett., 31, L12804, doi:10.1029/2004GL020192, 2004.

Lennartsson, W.: A scenario for solar wind penetration of Earth's magnetic tail based on ion composition data from the ISEE 1 spacecraft, J. Geophys. Res., 97, 19221-19238, 1992.

Lennartsson, O. W.: ISEE ion composition data with implications for solar wind entry into Earth's magnetotail, Space Sci. Rev., 80, 305-323, 1997.

Lennartsson, O. W.: In situ Polar observation of transverse coldion acceleration: Evidence that electric field generation is a hot-ion finite gyroradii effect, J. Geophys. Res., 108(A4), 1152, doi:10.1029/2002JA009663, 2003.

Lennartsson, O. W.: Auroral hot-ion dynamo model with finite gyroradii, Phys. Plasmas, 13, 072901, doi:10.1063/I.2217353, 2006.

Lennartsson, O. W., Trattner, K. J., Collin, H. L., and Peterson, W. K.: Polar/Toroidal Imaging Mass-Angle Spectrograph survey of earthward field-aligned proton flows from the near-midnight tail, J. Geophys. Res., 106, 5859-5871, 2001.

Lennartsson, O. W., Kistler, L., and Rème, H.: Plasma sheet fine structure at high latitude, Geophys. Res. Lett., 34, L18103, doi:10.1029/2007GL030753, 2007.

Lui, A. T. Y., Hones Jr., E. W., Venkatesan, D., Akasofu, S.-I., and Bame, S. J.: Complete plasma dropouts at Vela satellites during thinning of the plasma sheet, J. Geophys. Res., 80, 4649-4659, 1975.

Lui, A. T. Y., Zheng, Y., Rème, H., Dunlop, M. W., Gustafsson, G., and Owen, C. J.: Breakdown of the frozen-in condition in the Earth's magnetotail, J. Geophys. Res., 112, A04215, doi:10.1029/2006JA012000, 2007.

Mozer, F. S., Hudson, N. K., Torbert, R. B., Parady, B., and Yatteau, J.: Observations of paired electrostatic shocks in the polar magnetosphere, Phys. Rev. Lett., 38, 292-295, 1977.
Nakamura, R., Baumjohann, W., Mouikis, C., et al.: Spatial scale of high-speed flows in the plasma sheet observed by Cluster, Geophys. Res. Lett., 31, L09804, doi:10.1029/2004GL019558, 2004.

Ohtani, S.-I., Shay, M. A., and Mukai, T.: Temporal structure of the fast convective flow in the plasma sheet: Comparison between observations and two-fluid simulations, J. Geophys. Res., 109, A03210, doi:10.1029/2003JA010002, 2004.

Orsini, S., Candidi, M., Stokholm, M., and Balsiger, H.: Injection of ionospheric ions into the plasma sheet, J. Geophys. Res., 95, 7915-7928, 1990.

Parks, G. K., Lin, C. S., Anderson, K. A., Lin, R. P., and Reme, H.: ISEE 1 and 2 particle observations of outer plasma sheet boundary, J. Geophys. Res., 84, 6471-6476, 1979.

Parks, G. K., McCarthy, M., Fitzenreiter, R. J., et al.: Particle and field characteristics of the high-latitude plasma sheet boundary layer, J. Geophys. Res., 89, 8885-8906, 1984.

Petrukovich, A. A., Baumjohann, W., Nakamura, R., Schödel, R., and Mukai, T.: Are earthward bursty bulk flows convective or field-aligned?, J. Geophys. Res., 106, 21211-21215, 2001.

Pontius, D. H. and Wolf, R. A.: Transient flux tubes in the terrestrial magnetosphere, Geophys. Res. Lett., 17, 49-52, 1990.

Raj, A., Phan, T., Lin, R. P., and Angelopoulos, V.: Wind survey of high-speed bulk flows and field-aligned beams in the near-Earth plasma sheet, J. Geophys. Res., 107(A12), 1419, doi:10.1029/2001JA007547, 2002.

Rème, H., Aoustin, C., Bosqued, J. M., et al.: First multispacecraft ion measurements in and near the Earths magnetosphere with the identical Cluster ion spectrometry (CIS) experiment, Ann. Geophys., 19, 1303-1354, 2001, http://www.ann-geophys.net/19/1303/2001/.

Ruohoniemi, J. M. and Baker, K. B.: Large-scale imaging of highlatitude convection with Super Dual Auroral Radar Network HF radar observations, J. Geophys. Res., 103, 20797-20811, 1998.

Sauvaud, J.-A. and Kovrazhkin, R. A.: Two types of energydispersed ion structures at the plasma sheet boundary, J. Geophys. Res., 109, A12213, doi:10.1029/2003JA010333, 2004.

Sergeev, V. A., Sauvaud, J.-A., Popescu, D., et al.: Multiplespacecraft observation of a narrow transient plasma jet in the Earth's plasma sheet, Geophys. Res. Lett., 27, 851-854, 2000a.

Sergeev, V. A., Sauvaud, J.-A., Popescu, D., et al.: Plasma sheet ion injections into the auroral bulge: Correlative study of spacecraft and ground observations, J. Geophys. Res., 105, 18465-18481, 2000 b.

Spjeldvik, W. N. and Fritz, T. A.: Energetic ion and electron observations of the geomagnetic plasma sheet boundary layer: Threedimensional results from ISEE 1, J. Geophys. Res., 86, 24802486, 1981.

Tsyganenko, N. A.: Quantitative models of the magnetospheric magnetic field - Methods and results, Space Sci. Rev., 54, 75186, 1990.

Williams, D. J.: Energetic ion beams at the edge of the plasma sheet: ISEE 1 observations plus a simple explanatory model, J. Geophys. Res., 86, 5507-5518, 1981. 